\title{
MANIFOLD IMAGE PROCESSING FOR SEE-THROUGH EFFECT IN LAPAROSCOPIC SURGERIES
}

\author{
Sindhiya Devi.R ${ }^{1}$, Saravanaselvi.. ${ }^{2}$, Steena.J ${ }^{3}$ \\ ${ }^{1,3}$ II year M.E. student, ${ }^{2}$ Assistant Professor, Department of ECE, Einstein College of Engineering, Tirunelveli, \\ Tamilnadu, India
}

\begin{abstract}
This approach shows the processing of multiple images of a virtually transparent epidermal imagery (VTEI) system for LESS surgical procedure. In this paper, a novel Homographic Image Mosaicking and Morphing (HIMM) procedure is proposed for image-stitching and also for compensating the irregular surfaces, where scale invariant feature detection (SIFT) algorithm for detecting a set of feature points, vector field consensus (VFC) algorithm for mismatch rejection, Gauss Newton algorithm for non - linear minimization and backward image warping algorithms are used to obtain the see-through effect. This view is then noticeable to the surgeon in VTEI for laparoscopic surgeries.
\end{abstract}

Keywords: Biomedical devices, VTEI system, feature detection, mismatch rejection, image stitching

\section{INTRODUCTION}

Minimally Invasive Surgery (MIS), also called Laparoscopic surgery or keyhole surgery is a technique for surgeries in which the procedures are performed through tiny incisions in the abdomen (usually $0.5-1.5 \mathrm{~cm}$ ) rather than the larger incisions in open cavity surgeries. There are a variety of advantages of laparoscopic surgery over the open cavity surgical procedures. These include: Reduced hemorrhaging, smaller incision, reduced pain and shortened recovery time. However, this procedure is more difficult for the surgeon when compared to the traditional open surgery with limited range of motion, poor depth perception and difficult hand-eye correlation. The Minimally Invasive Surgical procedure also retain some challenges to surgeons which include restricted view and limited number of lookouts fixed by the insertion spots, an overhead screen that reveals the video from the videoscope but does not have a regular and rich indication of orientation of the video.

Minimally invasive surgery has become a standard of care for the medication of many benign and malignant gynaecological conditions. Laparoendoscopic single-site surgery (LESS), also known as single-port surgery, is a fresh and innovative, promptly onrushing minimally invasive technique. LESS enhances the aesthetic value of minimally invasive surgery by providing a single incision, where a trocar port can be inserted in the umbilicus. This appears to be a scar-free treatment.

This paper projects on the enhancement of LESS surgery with a Virtually Transparent Imagery (VTEI) system. It focuses on processing manifold imagery of the VTEI system.
After receiving the HD video signal from various sensor sources inside the body, image processing must be cautiously accomplished to provide a see-through effect for the surgeons. Various approaches [2]-[5] for visualization have been proposed to achieve "seeing through" effect. Image mosaicking is a well-studied research topic in computer vision [6] and has been widely applied in medical image analysis [7]-[10]. We present a novel Homographic Image Mosaicking and Morphing (HIMM) algorithm to stitch the images from various sensors to achieve a transparent system. Additionally, the proposed HIMM algorithm will alleviate the surgical tool overlay problem that is present in other approaches.

This paper is organized into four sections. Methodology is described in section II, the experimentation and the results are discussed in section III. Conclusion of this work is described in section IV.

\section{METHODOLOGY}

There are many rich attributes to be investigated before VTEI can become a reality in clinical assessments. Many surgeons have grown their comfortability towards HD-quality wired endoscopes.

The problem of using multiple cameras for image mosaicking is aggravated by the shape of the insufflated abdomen. Thus, we need a new hybrid stitching algorithm to replicate the perfect display. Hence we present a homographic image mosaicking and morphing algorithm to "focus" correctly on the concave surface of the abdomen. The wirelessly transmitted videos from two cameras from different viewing points are stitched together with partial overlapping areas to create a perfect panoramic video with high resolution. 
We designed a new image mosaicking method particularly for our VTEI system, which is when compared with conventional image mosaicking technique has some modifications such that we use the recently proposed vector field concensus (VFC) [11] instead of scale invariant feature transform (SIFT) [12] to reject mismatches.

To begin the HIMM procedure, we assume that there is a set of images transmitted to the computer from the cameras at any given time. These images will be mosaicked together. The procedure of HIMM is found in [6] can be summarized as follows: First, a set of SIFT feature points are detected in two adjacent images using the method which is presented in [12]. The points in the first image are denoted as xi, yi and the corresponding points in the second image as xi', yi'. The points between the two images satisfy the homography relationship between the images.To estimate the homographic matrix the error, E, must be minimized, which can be written as

$$
\min \mathrm{E}=\min \sum_{\mathrm{i}=1}^{\mathrm{n}}\left(\mathrm{x}_{\mathrm{i}}^{\prime \prime} \mathrm{x}_{\mathrm{i}}{ }^{\prime}\right)^{2}+\left(\mathrm{y}_{\mathrm{i}}^{\prime \prime} \mathrm{y}_{\mathrm{i}}^{\prime}\right)^{2}
$$

Since the relationship between (xi', yi',) and (xi,yi) is nonlinear, Gauss-Newton optimization is used to estimate the homography parameters. Once when the homography matrices are computed, we will be able to warp one image to another in a piecewise manner. First, a reference frame is selected and then all the other frames are warped back into the reference image's coordinate. Since forward mapping might create black seams for those pixels that are not mapped, we use the backward mapping incorporated with bilinear interpolation. The overlap area of the final composite image might look blurry. Hence we use simple feathering to solve this problem.

\section{RESULTS AND DISCUSSIONS}

In this paper, we present a novel HIMM algorithm for Homographic Image Mosaicking and Morphing to create a see-through effect of the surgical area for the surgeons.

Planar 2D images are projected directly onto the insufflated abdomen to give surgeons an open-cavity view of the surgical area. This projection over 2D images introduces a distortion effect that is disorienting for the surgeon. To overcome this effect, the mosaicking is combined with a morphing algorithm. This combination of mosaicking and morphing into a single algorithm is referred to as HIMM and it can be run in real-time.

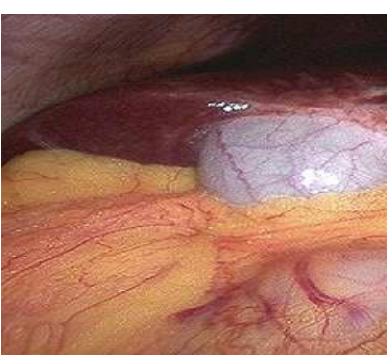

(a)

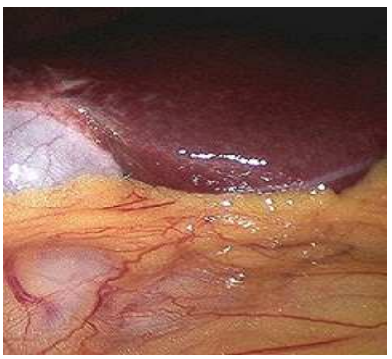

(b)

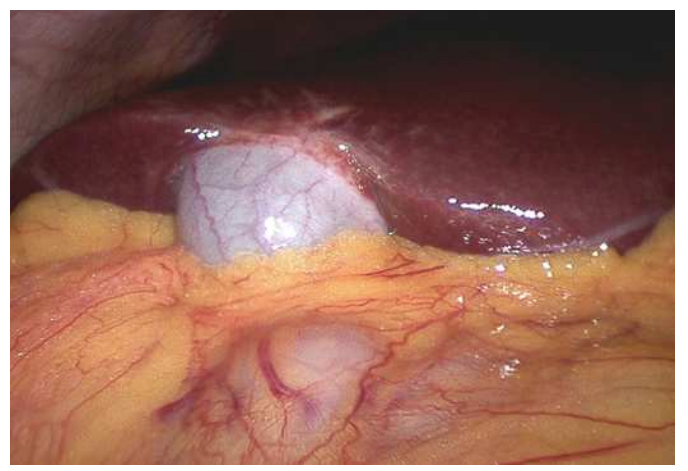

(c)

Fig -1: HIMM algorithm output. a)Image captured by the left camera, b) Image captured by the right camera, c) Image after fusion.

The images shown in fig 1(a) and fig 1(b) are obtained from two cameras in different views. The final blending result is shown in Fig. 1(c) where the original left and right images are perfectly mosaicked and morphed into a single display. Thus the HIMM algorithm can maintain a correspondence between the various inputs and also it can provide the open cavity view needed by the surgeons.

\section{CONCLUSIONS}

Next-generation biomedical devices for advanced minimally invasive surgeries will require wireless links to get data from in vivo image sensors. Our work deals with the process of image mosaicking and morphing by using a novel HIMM algorithm to create an open cavity view to the surgeons.

\section{REFERENCES}

[1]. Y. Sun, A. Anderson, C. Castro, B. Lin, and R. Gitlin, "Virtually transparent epidermal imagery for laparoendoscopic single-site surgery," in Proc. Int. Conf. IEEE Engineering in Medicine and Biology Soc., Aug.2011.

[2]. M. Blackwell, C. Nikou, A. Digioia, and T. Kanade, "An image overlay system for medical data 
visualization," Med. Image Anal., vol. 4, no. 1, pp. 6772, 2000.

[3]. H. Fuchs, M. A. Livingston, R. Raskar, D. Colucci, K. Keller, A. State, J. R. Crawford, P. Rademacher, S. H. Drake, and A. A. Meyer, "Augmented reality visualization for laparoscopic surgery," in Proc. Medical Image Computing and Computer Assisted Intervention Conf., 1998, pp.11-13.

[4]. H. Hoppe, G. Eggers, T. Heurich, J. Raczkowsky, R. Marmuller, H.Worn, S. Hassfeld, and L. Moctezuma, "Projector-based visualization for intraoperative navigation: First clinical results," in Proc. 17th Int.Congress and Exhibition on Computer Assisted Radiology and Surgery,2003, p. 771.

[5]. S. Nicolar, X. Pennec, L. Soler, and N. Ayache, "A complete augmented reality guidance system for liver punctures: First clinical evaluation," in Proc. Medical Image Computing and Computer Assisted Intervention Conf., 2005, pp. 539-547.

[6]. R. Szeliski, "Image alignment and stitching: A tutorial," Found. Trends.Comput. Graph. Vis., vol. 2, no. 1, pp. 1-104, Jan. 2006.

[7]. S. Seshamani, W. W. Lau, and G. D. Hager, "Real-time endoscopic mosaicking," in Proc. Medical Image Computing and Computer Assisted Intervention Conf., 2006, pp. 355-363.

[8]. W. Konen, M. Naderi, and M. Scholz, "Endoscopic image mosaics for real-time color video sequences," in Proc. Computer Assisted Radiology and Surgery, 2007.

[9]. P. C. Amd, C. H. Bay, L. V. Gool, and G. Szkely, "Retina mosaicing using local features," in Proc. Medical Image Computing and Computer Assisted Intervention Conf., 2006.

[10]. A. Kolar, "Prototype of video endoscopic capsule with 3-d imaging capabilities," IEEE Trans. Biomed. Circuits Syst., vol. 4, no. 4, pp.239-249, 2010.

[11]. J. Zhao, J. Ma, J. Tian, J. Ma, and D. Zhang, “A robust method for vector field learning with application to mismatch removing," in Proc.IEEE Conf. Computer Vision and Pattern Recognition, Jun. 2011, pp.29772984.

[12]. D. G. Lowe, "Distinctive image features from scaleinvariant key-points," Int. J. Comput. Vision, vol. 60, no. 2, pp. 91-110, Nov. 2004.

\section{BIOGRAPHIES}

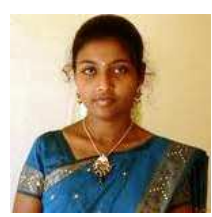

Sindhiya Devi.R received the B.E degree in 2010, through Anna University, Chennai, India and currently pursuing M.E in the same university. Her research interests include computer vision and medical image processing.

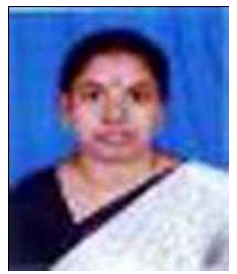

Saravanaselvi.P received the B.E and M.E degrees in 2001 and 2009 respectively through Anna University, Chennai, India. Currently, she is an Assistant Professor in Einstein College of Engineering, Tirunelveli, India. Her research interests include computer vision.

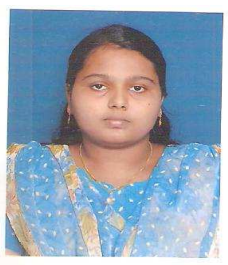

Steena.J received the B.E degree in 2012 through Anna University, Chennai, India and she is currently pursuing M.E in the same university. Her research interests include computer vision. 